International Math Olympiad, all while still a high school student. He was also on Harvard's winning Putnam team in 2011 and a Putnam Fellow in 2012. Eric is one of the most accomplished students of mathematics that the mathematics community has ever seen. In the words of Ken Ono, "Eric is a phenomenon."

\section{Biographical Sketch}

Eric Larson is a graduate student at MIT in mathematics. He is from Eugene, Oregon, where, while in elementary school, he discovered his love of mathematics after seeing Euclid's proof of the infinitude of primes. Eric received his bachelor's degree in mathematics from Harvard University, with a secondary in physics. His research interests are concentrated in algebraic geometry and number theory. Currently, he is working on a couple of projects related to the geometry of general curves in projective space.

\section{Response from Eric Larson}

I am honored to receive the 2014 Frank and Brennie Morgan Prize for Outstanding Research in Mathematics by an Undergraduate Student, and would like to warmly thank AMS, MAA, and SIAM.

I am also grateful to the many people that have helped me get here. Especially, I would like to thank my research mentors Ken Ono, Joe Harris, and David Zureick-Brown, as well as my family and friends for their support and encouragement.

\section{About the Prize}

The Morgan Prize is awarded annually for outstanding research in mathematics by an undergraduate student (or students having submitted joint work). Students in Canada, Mexico, or the United States or its possessions are eligible for consideration for the prize. Established in 1995, the prize was endowed by Mrs. Frank (Brennie) Morgan of Allentown, Pennsylvania, and carries the name of her late husband. The prize is given jointly by the AMS, the Mathematical Association of America (MAA), and the Society for Industrial and Applied Mathematics (SIAM) and carries a cash award of US\$1,200.

Recipients of the Morgan Prize are chosen by a joint AMS-MAA-SIAM selection committee. For the 2013 prize, the members of the selection committee were Colin C. Adams, Bela Bajnok, Johnny Guzman, Kathleen R. Fowler, Reza Malek Madani, and Susan E. Martonosi.

Previous recipients of the Morgan Prize are Kannan Soundararajan (1995), Manjul Bhargava (1996), Jade Vinson (1997), Daniel Biss (1998), Sean McLaughlin (1999), Jacob Lurie (2000), Ciprian Manolescu (2001), Joshua Greene (2002), Melanie Wood (2003), Reid Barton (2005), Jacob Fox (2006), Daniel Kane (2007), Nathan Kaplan (2008), Aaron Pixton (2009), Scott Duke Kominers (2010), Maria Monks (2011), John Pardon (2012), and Fan Wei (2013).

-Elaine Kehoe

\title{
2014 Conant Prize
}

Alex Kontorovich was awarded the 2014 Levi L. Conant Prize at the 120th Annual Meeting of the AMS in Baltimore, Maryland, in January 2014.

\section{Citation}

The 2014 Levi L. Conant Prize is awarded to Alex Kontorovich for his article, "From Apollonius to Zaremba: Local-global phenomena in thin orbits", Bulletin of the American Mathematical Society 50 (2013), no. 2, 187-228.

This article introduces us to a new field of number theory that has proven to be extremely fruitful, even in shedding light on some ancient problems. The author illustrates the new ideas by focusing on three problems, which at first glance seem totally unrelated, but each of which is an attractive mixture of algebra and geometry. The first problem (Zaremba's Conjecture) asks whether every integer DOI: http://dx.doi.org/10.1090/noti1109 is the denominator of a fraction which can be expressed as a continued fraction

$$
x=\frac{1}{a_{1}+\frac{1}{a_{2}+\ldots}},
$$

where the $a^{j}$ are constrained to be $1,2,3,4,5$. The second problem is more overtly geometric and asks whether all sufficiently large integers (not prohibited by congruence conditions) occur as curvatures in an integral Apollonian gasket, a configuration of circles with many tangencies. Finally, the third problem asks if there are infinitely many primes that occur as hypotenuses in a thin orbit of Pythagorean triples.

Kontorovich masterfully introduces the general reader to these problems and the ways in which they are connected through the concept of orbits of groups of matrices that are of infinite index in a 


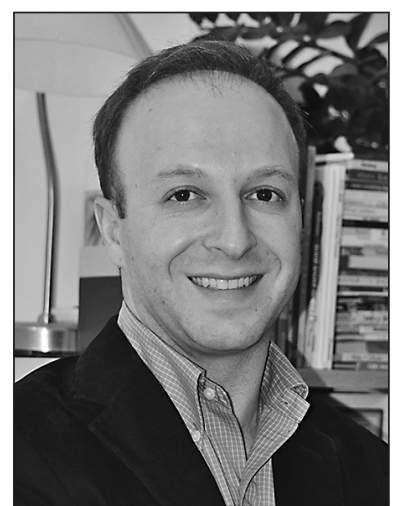

Alex Kontorovich group of integral matrices (hence the "thin"). He also introduces the "circle method", a heuristic principle for going from local to global results.

The article is full of pictures, interesting history, and references to a great deal of mathematics, some very classical and some very modern. As the author points out, it is a side of number

theory that borrows freely from $L$-functions, the Langlands program, and classical sieve theory as well as a number of other fields without being subsumed by any of them.

The article dips into some deep mathematics while maintaining a casual flow so that both expert and novice readers alike are entertained throughout this look at ancient problems through a modern lens.

\section{Biographical Sketch}

Alex Kontorovich is an assistant professor of mathematics at Yale University. He was born in 1980 in Voronezh, Russia, and grew up in New Jersey after the family emigrated in 1988 . He received a B.A. from Princeton in 2002 and a Ph.D. in 2007 from Columbia, advised by Dorian Goldfeld and Peter Sarnak. Following a Tamarkin Assistant Professorship at Brown (2007-2010), he taught at Stony Brook (2010-2011) before moving to Yale. He is the recipient of an NSF Postdoctoral Fellowship, an NSF CAREER Award, and a Sloan Research Fellowship, and has twice been a year-long member at the Institute for Advanced Study. His other joys include music and spending time with his family, wife Amy and son Harry.

\section{Response from Alex Kontorovich}

I am deeply humbled and very surprised to receive the 2014 Levi L. Conant Prize from the American Mathematical Society. The idea of writing an expository article had never occurred to me until Andrew Granville planted the seed in my head years ago. Over time, Andrew gently prodded until I finished the task, making innumerable comments and suggestions which drastically improved various drafts along the way (of course his writings were my model of outstanding exposition); a share of the prize belongs to him. Another share belongs to Jean Bourgain: the main theorems discussed in this paper are part of our ongoing collaboration, and I am grateful for his tutelage. There would have been nothing to report had Peter Sarnak not introduced us to Apollonian gaskets, and Curt
McMullen to Zaremba's conjecture. I should also like to take this opportunity to thank my many teachers. In addition to the more obvious (advisors and co-authors). these include Yakov Sinai, Eli Stein, John Conway, John Morgan, and Ioannis Karatzas. I greatly enjoyed the opportunity and challenge to collect some aspects of thin groups in this paper; hopefully, it might encourage more people to go into this new field.

\section{About the Prize}

The Conant Prize is awarded annually to recognize an outstanding expository paper published in either the Notices of the AMS or the Bulletin of the $A M S$ in the preceding five years.

Established in 2001, the prize honors the memory of Levi L. Conant (1857-1916), who was a mathematician at Worcester Polytechnic Institute. The prize carries a cash award of US\$1,000.

The Conant Prize is awarded by the AMS Council acting on the recommendation of a selection committee. For the 2013 prize, the members of the selection committee were Thomas F. Banchoff, Brian Conrey, and John F. Oprea.

Previous recipients of the Conant Prize are: Carl Pomerance (2001); Elliott Lieb and Jakob Yngvason (2002); Nicholas Katz and Peter Sarnak (2003); Noam D. Elkies (2004); Allen Knutson and Terence Tao (2005); Ronald M. Solomon (2006); Jeffrey Weeks (2007); J. Brian Conrey, Shlomo Hoory, Nathan Linial, and Avi Wigderson (2008), John W. Morgan (2009), Bryna Kra (2010), David Vogan (2011), Persi Diaconis (2012), and John Baez and John Huerta (2013).

-Elaine Kehoe 